\title{
Fragmentation of a Protostellar Core: The Case of CB 230
}

\author{
Ralf Launhardt \\ Caltech, Astronomy Dept., MS 105-24, Pasadena, CA 91125, USA
}

\begin{abstract}
The Bok globule CB 230 (L 1177) contains an active, lowmass star-forming core which is associated with a double NIR reflection nebula, a collimated bipolar molecular outflow, and strong $\mathrm{mm}$ continuum emission. The morphology of the NIR nebula suggests the presence of a deeply embedded, wide binary protostellar system. High-angular resolution observations now reveal the presence of two sub-cores, two distinct outflow centers, and an embedded accretion disk associated with the western bipolar NIR nebula. Judging from the separation and specific angular momentum, the CB 230 double protostar system probably results from core fragmentation and will end up at the upper end of the pre-main sequence binary separation distribution.
\end{abstract}

\section{Motivation and Observations}

Binary systems have been observed in all pre-main-sequence stages of evolution and there is growing evidence for proto-binary systems, although the numbers are still small (Mundy, this volume). Both theory and observations support the hypothesis that most binary systems form by fragmentation during the gravitational collapse of molecular cloud cores. We have started a program of high angular resolution observations to study the early formation process of binary stars in detail, using the Owens Valley Radio Observatory (OVRO) millimeter array (Launhardt, Sargent, and Zinnecker 2000).

This paper presents new observations of the protostellar core in the Bok globule CB 230 ( $D=450 \mathrm{pc}$ ). CB 230 contains a dense core (Launhardt et al. 1997, 1998, 2000) and two associated NIR reflection nebulae separated by $\sim 10^{\prime \prime}$ (Yun 1996; Launhardt 1996, Fig.1). The western nebula is bipolar, with a bright northern lobe perfectly aligned with the blue lobe of a collimated CO outflow (cf. Yun \& Clemens 1994, Fig.3). The eastern nebula is much fainter and redder and displays no bipolar structure. No associated stars are visible.

CB 230 was observed at 1.2 and $3 \mathrm{~mm}$ with the OVRO $\mathrm{mm}$ array in spring 2000. The mm continuum emission traced the optically thin thermal dust emission, while molecular gas was traced by the $\mathrm{N}_{2} \mathrm{H}^{+}(1-0)$ and ${ }^{13} \mathrm{CO}(1-0)$ lines at 93 and at $110 \mathrm{GHz}$, respectively. $\mathrm{N}_{2} \mathrm{H}^{+}(1-0)$ comprises seven hyperfine components and, compared to other molecules, depletes later and more slowly onto grains (Bergin \& Langer 1997). It is a reliable indicator of the morphology and kinematics of protostellar cores. ${ }^{13} \mathrm{CO}$ traces the small-scale structure of the outflow near the driving sources. Beam sizes for the different observations vary from $1^{\prime \prime}$ to $10^{\prime \prime}$, and the spectral resolution was $\sim 0.2 \mathrm{~km} / \mathrm{s}$. 

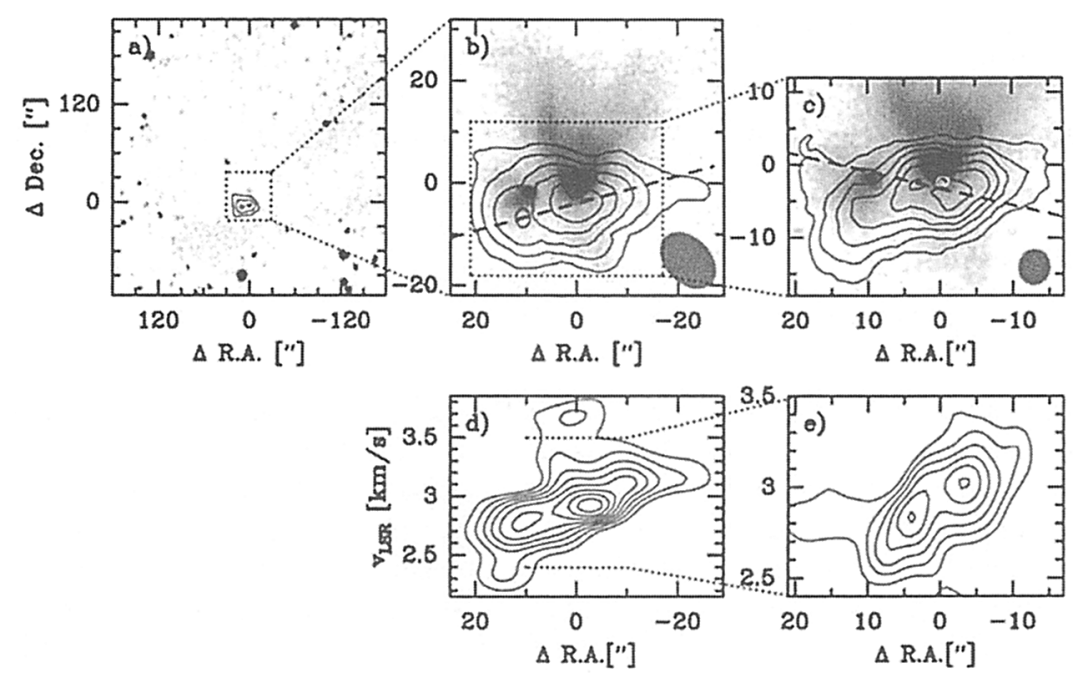

Figure 1. OVRO $\mathrm{N}_{2} \mathrm{H}^{+}(1-0)$ observations of the CB 230 double core. a) Optical image (DSS) overlayed with $\mathrm{N}_{2} \mathrm{H}^{+}$contours. b) K-band image of the NIR nebulae overlayed with the intensity map of the $\mathrm{N}_{2} \mathrm{H}^{+}(12-01)$ triplet. c) As b) but at $4.5^{\prime \prime}$ resolution. White contours show the $1 \mathrm{~mm}$ continuum emission (cf. Fig.2). All contours start with $3 \sigma$. d) and e) Position-velocity diagrams of the $\mathrm{N}_{2} \mathrm{H}^{+}(123-$ 012 ) transition along PA indicated by the dashed lines in Figs. b) and c).

\section{Results}

\subsection{Morphology and Kinematics of the Double Core}

The NIR morphology of CB 230 suggests the presence of two deeply embedded YSO's separated by $\sim 10^{\prime \prime}$ (Fig. 1b). These were not resolved in previous singledish $\mathrm{mm}$ observations. However, with higher angular resolution observations and using an optically thin line which is unlikely to deplete in protostellar cores $\left(\mathrm{N}_{2} \mathrm{H}^{+}(1-0)\right)$, the object can be resolved.

At $9^{\prime \prime}$ resolution, the $\mathrm{N}_{2} \mathrm{H}^{+}$maps show that the molecular cloud core is elongated E-W and consists of two sub-cores separated in space $\left(10^{\prime \prime} \simeq 4500 \mathrm{AU}\right)$ and velocity. Each sub-core coincides spatially with one of the two NIR nebulae (Fig. 1b). The velocity field and the position-velocity (p-v) diagram along the major axis indicate that the double core rotates with $\omega \sim 2 \times 10^{-13} \mathrm{~s}^{-1}$ around an axis perpendicular to connecting line of the sub-cores and approximately parallel to the large-scale outflow axis (Figs. 1b and d). At higher angular resolution $\left(4.5^{\prime \prime}\right)$, the stronger western sub-core splits up into two separate maxima located almost symmetric to the mm continuum source at the origin of the western outflow and NIR nebula (Fig.1c). Since there is also a large velocity gradient along this double-peaked western sub-core, it can be interpreted as a thick gaseous disk or torus with $R \sim 1600 \mathrm{AU}$, rotating at a rate of $\omega \sim 5 \times 10^{-13} \mathrm{~s}^{-1}$ around the embedded accretion disk (see Sect.2.2). Despite its smaller size, this torus has about the same specific angular momentum as the entire cloud core, $J / M \sim 5 \times 10^{-17} \mathrm{pc}^{2} \mathrm{~s}^{-1}$, suggesting that it has rotationally decoupled from the cloud core. 


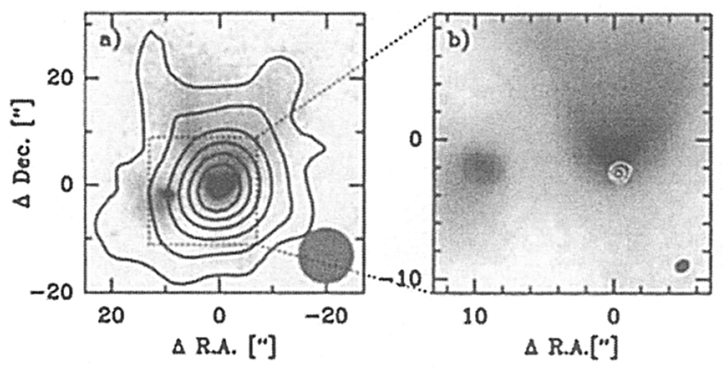

Figure 2. $\quad 1.3 \mathrm{~mm}$ dust continuum emission from CB 230. a) K-band image of the NIR reflection nebula (grey-scale) overlayed with contours of the $1.3 \mathrm{~mm}$ continuum emission (IRAM 30-m; $1 \sigma=7 \mathrm{mJy} /$ beam; levels $20,40,75,105, \ldots$ mJy/beam). b) Enlarged K-band image overlayed with the $1 \mathrm{~mm}$ continuum emission from OVRO $(1 \sigma=3.6 \mathrm{mJy} /$ beam; levels $11,22,33 \mathrm{mJy} /$ beam $)$.

\subsection{Dust Continuum Emission from Embedded Disk(s)}

Strong $1 \mathrm{~mm}$ continuum emission peaks at the origin of the western bipolar NIR nebula (Fig. 2a). This dust emission is extended towards the eastern NIR nebula. The total mass of the CB 230 core implied is $7 \mathrm{M}_{\odot}$, of which $\sim 2 \mathrm{M}_{\odot}$ can be attributed to the unresolved central condensation (Launhardt et al. 2000). Figure 2a explains the north-south asymmetry of the western bipolar nebula; the southern (redshifted) lobe is heavily obscured by dust in the dense core, while in the nortern (blueshifted) lobe we are looking directly into the outflow cone. The OVRO $1 \mathrm{~mm}$ dust continuum map shows a compact source associated with the origin of the western bipolar nebula and outflow (Fig.2b). It is unresolved in the $1^{\prime \prime}$ beam, suggesting the presence of an embedded accretion disk with radius $R<200 \mathrm{AU}$ and mass $M \sim 0.1 M_{\odot}$. A significant contribution by freefree emission can be ruled out since the bolometric luminosity of the entire cloud core of $11 \mathrm{~L}_{\odot}$ points to a low-mass protostar with no capability to ionize its environment (Launhardt et al. 1997). The eastern source may be too faint to detect $\left(<2 \mathrm{mJy}\right.$ at $3 \mathrm{~mm}$ and $<10 \mathrm{mJy}$ at $\left.1 \mathrm{~mm} ; M<0.006 M_{\odot}\right)$ or simply lack a disk.

\subsection{The Outflow is also Double}

The OVRO ${ }^{13} \mathrm{CO}(1-0)$ map of $\mathrm{CB} 230$ at $4^{\prime \prime}$ resolution reveals that the largescale outflow discovered by Yun \& Clemens (1994) is actually composed of two approximately co-oriented pairs of lobes. The outflow centers are $\sim 10^{\prime \prime}$ apart and coincide with the assumed origins of the two NIR reflection nebulae, thus confirming the presence of two embedded protostars (Fig. 3). The outflow velocities are small $\left(\Delta v_{\text {mean }} \sim 0.7 \mathrm{~km} / \mathrm{s}, \Delta v_{\max } \sim 1 \mathrm{~km} / \mathrm{s}\right)$ and the exact alignment cannot be derived since the southern, red lobes overlap. The extended ${ }^{13} \mathrm{CO}$ gas at the rest velocity of the cloud core $\left(v_{\mathrm{LSR}}=2.9 \pm 0.2 \mathrm{~km} / \mathrm{s}\right)$ is completely resolved out in the interferometer image. 


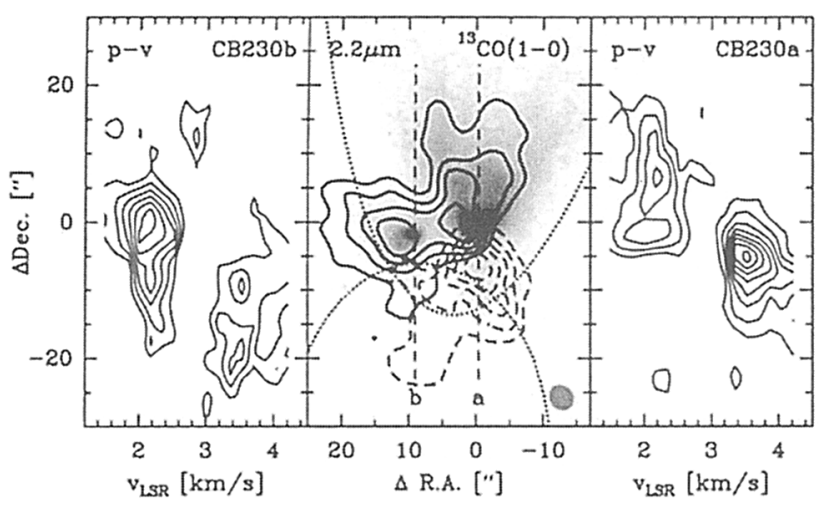

Figure 3. OVRO ${ }^{13} \mathrm{CO}(1-0)$ observations of the CB 230 outflow. Middle panel: $\mathrm{K}$-band image of the NIR nebula overlayed with channel maps of the blue and red ${ }^{13} \mathrm{CO}(1-0)$ outflow lobes. Blue lobe: $v_{\mathrm{LSR}}=1.6 \ldots 2.8 \mathrm{~km} / \mathrm{s}$, solid contours. Red lobe: $v_{\mathrm{LSR}}=3.0 \ldots 4.2 \mathrm{~km} / \mathrm{s}$, dashed contours. Contours start at $3 \sigma$. The dotted ellipses outline the large-scale ${ }^{12} \mathrm{CO}$ outflow mapped by Yun \& Clemens (1994; HPBW $48^{\prime \prime}$ ). Right and left panels: P-V diagrams for sources CB $230 \mathrm{a}$ and $\mathrm{b}$ along the slices indicated by dashed lines in the middle panel.

\section{CB 230 in the Framework of Binary Star Formation}

The globule CB 230 contains an embedded, wide binary protostellar system with a projected separation of $\sim 4500 \mathrm{AU}$, placing the system at the upper end of the pre-main sequence binary separation distribution (Mathieu 1994). The specific angular momentum of the double core is $J / M \sim 5 \times 10^{-17} \mathrm{pc}^{2} \mathrm{~s}^{-1}$, which is at the lower end of values for dense cores in dark clouds (e.g., Goodman et al. 1993), but still at the upper end of the range for pre-main sequence binary star systems (Simon et al. 1995). In this sense, the CB 230 system closes the gap between pre-star-forming molecular cloud cores and young binary star systems. The ratio of rotational kinetic to gravitational energy of the double core is $\beta \sim 0.01$. This value is too small to explain the binary formation due to pure rotational breakup, but large enough to be consistent with fragmentation of a slowly rotating, magnetically supported cloud (Bodenheimer, Boss, Klein, this volume).

\section{References}

Bergin, E. A., \& Langer, W. D. 1997, ApJ, 486, 316

Goodman, A. A., Benson, P. J., Fuller, G. A., \& Myers, P. C 1993, ApJ, 406, 528

Launhardt, R. 1996, PhD thesis, University of Jena

Launhardt, R., \& Henning, Th. 1997, A\&A, 326, 329

Launhardt, R., Evans II, N. J., Wang J., et al. 1997, ApJS, 119, 59

Launhardt, R. Ward-Thompson, D., \& Henning Th. 1998, MNRAS, 288, L45

Launhardt, R., Henning, Th., \& Zylka, R. 2000, in preparation

Launhardt, R., Sargent, A., \& Zinnecker, H. 2000, Proc. ALMA conf., in press 
Launhardt, R., Sargent, A., \& Zinnecker, H. 2000, Sience with the Atacama Large Millimeter Array, ed. A. Wootten, ASP Conf. Series, in press Mathieu, R. D. 1994, ARA\&A, 32, 465

Simon, M., Ghez, A. M., Leinert, Ch., et al. 1995, ApJ, 443, 625

Yun, J. L. \& Clemens, D. P. 1994, ApJS, 92, 145

Yun, J. L. 1996, AJ, 111, 930

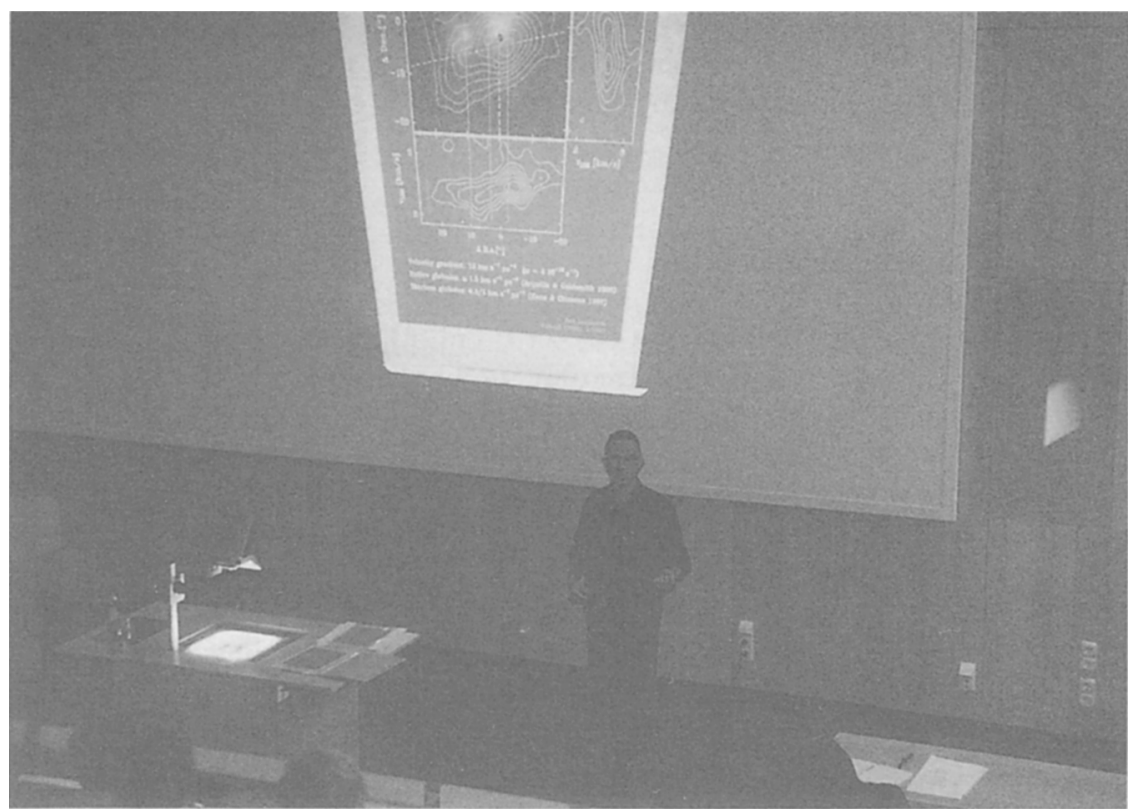

Ralf Launhardt 